\title{
Fpga Based Optimal Charging In a Solar Powered Robot
}

\author{
M. Ragulkumar ${ }^{1}$, P. Manikandan ${ }^{2}$, Dr G. K. D Prasanna Venkatesan ${ }^{3}$, \\ Post Graduate Student- ME VLSI Design ${ }^{1}$, Assistant Professor ${ }^{2}$, Vice Principal, Professor and Head \\ Department of ECE., PGP College of Engineering Technology, Namakkal, Tamilnadu, India.
}

\begin{abstract}
FPGA Robotics is commonly used in a VANTER Robotics and space. An efficient solar power FPGA Robotic designs contains low power consumption and improved batteries life. The advantage of a rover in which most of the supplied energy is reduced by a photo voltaic $(P V)$ panel. However the solar panel powered rover introduces the concept of using FPGA for intelligent power management system. The rover has assisted coupled an assisted suspension mechanism this prevents a manipulator arm the rover from having to minimize solar panel generated power and allows it to dust solar panel surface. The robot uses a control algorithm of maximum power point (MPP) aimed at maximizing system supplied power for PV modules designs of its mechanical structure and its simulation results are presented by ModelSim.
\end{abstract}

Key words: Field Programmable Gate Array (FPGA), VANTER, Photo voltaic (PV) panel, Rover, MPPT.

\section{Introduction:}

The energy management system and solar tracking mechanism is one of the significant and crucial factors in robotic platform. The solar tracking mechanism and power system performance by pack of two Li-Po batteries are tested on VANTER Robotic Platform. Optimization charging implemented to increase rover power, to charging and discharging [1]. The system-on-a-programmable chip (SoPC) technology and hardware/software code design technique are implemented into Field Programming Gate Array (FPGA) chip to achieve stabilization, trajectory tracking, parameter variation, uncertainty and path following [2]. In this literature, optimal battery charging is achieved by Voltage based Maximum Power Point Tracking (VMPPT) technique using microprocessor within shorter duration. The operating point adjusted by changing the charging current. But FPGA processor is stable also in maximum power point [3]. This MPPT technique in Photo Voltaic (PV) increases the PV output power using microcontroller. However FPGA MPPT control algorithm automatically adjusts changes in external environment [4].

A Kinematic model of robot, the family feasible trajectory and their corresponding steering control are derived in a closed form and are expressed terms of one adjustable parameter which consist of a time criterion and geometrical criterion [5]. Robotic exploration depends on efficient power resources. Robotic planner predicts shadowing and solar power generation for nearby location suitable for recharging [6].

The first invention of rechargeable battery in space mission is Mars Exploration Rovers. Nevertheless the need for need for greater operation autonomy spirit and opportunity was solved by means larger deploy solar panels [7].Redesigning with specific aspects of the some preliminary result of a new rover concept to develop a system design for a regional exploration rover including bread boarding for demonstration of locomotion capabilities, payload accommodation, power provision and control [8].The method of DC power generation by fixed solar cells and to energize LED light sources operated by directly connected white LED with current limitation [9].

In the present, the optimal charging is directly performed through software programmed using Verilog Hardware Description Language (VHDL) codes in Xilinx ISE 8.1. The FPGA technology offers a fast system with many more advantages as compared to other conventional technology including Microcontroller based controller. The software programmed can easily optimized battery charging and power system management.

\section{Robotic Platform:}

The VANTER robotic exploration vehicle aims to improve various aspects of the aforementioned rovers with scientific and academic purposes. The rover was developed and guided to have a set of four wheels coupled to a plane chassis that can rotate independently. The four-wheel-drive and the individual control of each wheel allow different types of movement: including control of each wheel allow different types of movement; including the rover movement and optimal charging. The four wheels in VANTER robot are sustained by means of independent passive suspension of double aluminuium fork to absorb substain vibrations shown in Fig [1].

Each wheel consists of single motor it used for as rotation and movement of robot. On the one hand, forward movement is produced by means of dc a motor that provides $120 \mathrm{r} / \mathrm{min}$ with a torque of 8.87 
$\mathrm{kg} / \mathrm{cm}$. on the other the rotation motor provides a speed of $152 \mathrm{r} / \mathrm{min}$. The robotic vehicle having single tilt able Solar PV Panels it can be controlled by means of solar tracked panels.

The robotic system programming is divided into two main code levels and its hardware was designed with a hierarchical control structure based on modular controllers. The top level program, carried out in VHDL language, is executed in a remote PC and offers a FPGA Technology to monitor and control the whole robotic vehicle.

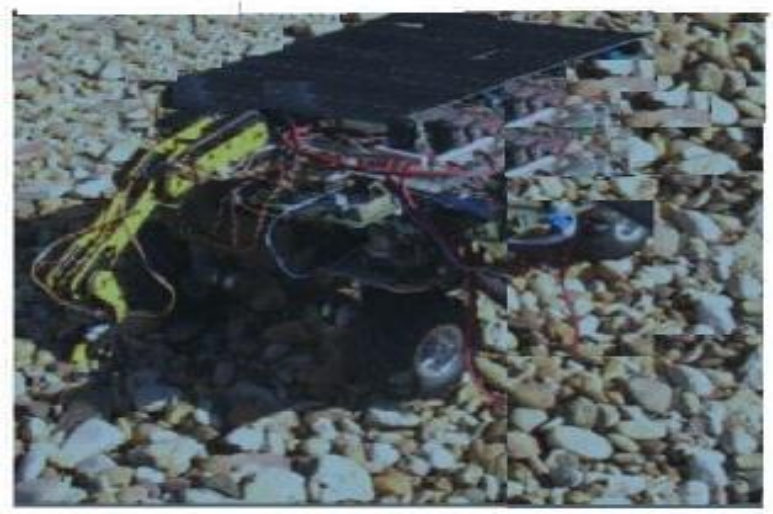

Fig.1. VANTER: A solar-powered robotic vehicle

\section{Fpga Technology:}

FPGAs have established themselves as the most flexible and reconfigurable intelligence in applications from networking and Telecommunications equipment to industrial and automotive segments. More recently FPGAs have proliferated into consumer devices such as set-top boxes and few more electronic devices. But future growth in FPGA usage will come from their adoption into portable devices such as GPS, medical, instrumentation, and consumer devices. Multiple voltages are required to power an FPGA: Core voltage $(0.9 \mathrm{~V}$ to $2.5 \mathrm{~V})$, I/O voltage $(2.5 \mathrm{~V}$ to $3.3 \mathrm{~V})$ and another low-noise, low-ripple voltage for auxiliary circuits $(2.5 \mathrm{~V}$ or $3.3 \mathrm{~V})$. Furthermore, when FPGAs are operates from batteries, the system efficiency and battery life becomes very crucial. A power management with a special focuses on portable systems, are:

- System-level challenges in powering FPGAs in portable devices

- Monotonic rise of core voltage

- Voltage scaling and back body biasing is used to improve FPGAs efficiency.

In portable devices, efficiency during both active and stand-by modes of operation is of paramount importance. Efficiency directly affects battery life and the duration of usable operation. The input voltages for battery-powered systems range anywhere from $1.8 \mathrm{~V}$ to $5.5 \mathrm{~V}$. The most common sources of power are either 2 AA or single Li-Ion cell batteries whose voltage ranges from $3 \mathrm{~V}$ to $4.2 \mathrm{~V}$. Operating currents are typically less than $1.5 \mathrm{~A}$, with most applications requiring less than $600 \mathrm{~mA}$. Although there are general guidelines for using the right step-down solution for powering, portable devices mandate that high efficiency be maintained even during standby to extend battery life.

FPGAs are essentially CMOS devices, which scale with advances in process technology. As device geometries scale below $90 \mathrm{~nm}$ and operating frequencies increase, both dynamic and static power consumption become more important. Current approaches to FPGA design do not allowed for easy reduction of dynamic or static power, although it possible to theoretically. To further improve efficiency significantly, techniques such as adaptive voltage scaling should be implemented. As devices scale downward of $90 \mathrm{~nm}$, managing static power becomes equally important due to the rise in leakage currents in the ICs.

The use of innovative back-biasing power management ICs is suggested to reduce sub-threshold leakage and static power. Furthermore, pre-biased loads may exist on the FPGAs during start-up. To ensure monotonic rise of the core voltage is important for their proper functioning, power management ICs that start-up properly into pre-biased loads should be used. In order to comparison the controller implementations of FPGAs offer lower cost implementations since the functions of various components can be integrated onto the same FPGA chip.

In addition, FPGAs can provide equivalent or higher performance. Because FPGAs can be reprogrammed at any time, repairs can be performed in-site while the system is running thus providing a high degree of robustness. 


\section{a. Wheeled Mobile Robot:}

\section{Robotic System Design:}

A robot capable of locomotion on a surface solely through the actuation of wheel assemblies mounted on the robot and in contact with the surface. A wheel assembly is a device which provides or allows relative motion between its mount and a surface on which it is intended to have a single point of rolling contact. Wheeled robots comprise one or more driven wheels and possibly steered wheels. Most designs require two motors for driving a mobile robot. The design is also steered. It requires two motors; one for driving the wheel on the left-hand side of Figure 1 has a single driven wheel that and another one for turning. The advantage of this design is the driving and turning actions have been completely separated by using two different motors. b. Architecture of FPGA Robot:

The architecture of FPGA controller to interconnects for the Battery monitoring and Motion control and Light sensor. The ADC Converter is used for to convert analog value to digital value. The sensor has connected to the driver circuit. This circuit has to connect for analog signal to convert digital values. The battery system used for charging and discharging, monitoring the battery system.

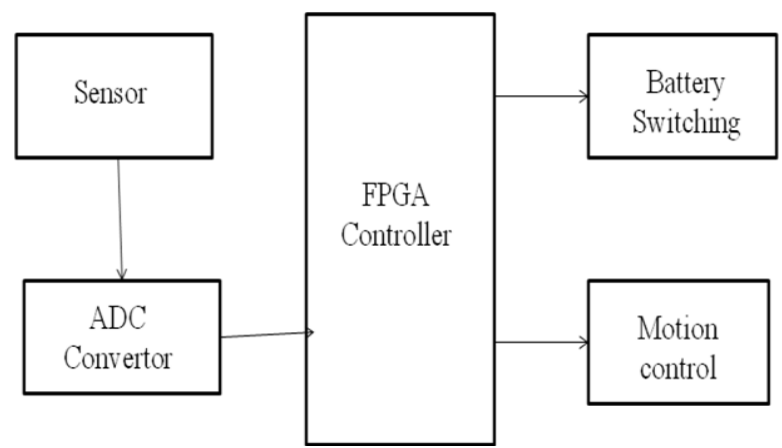

Fig.2 Block Diagram of FPGA Robot.

\section{c. Battery System:}

There are three cases in the proposed Li-Po battery charger. It is a slight difference in remaining capacity between the two Batteries, B1 is almost identical to B2; another is B1 smaller than B2; the other is B1large than B2. By means of the sensed B1and B2 and the proposed charging strategy, charge equalization between battery 1 and battery 2 can be achieved via energy recycling. The proposed charger topology is shown in Fig.3.

\section{d. Optimal Battery Charging:}

The charging and discharging makes the two fold significant configuration. The process of charging a battery independently performed while the other battery provides all the energy consumed by the robotic vehicle. The charge battery obtained from the PV panels is routed to the charger and from the charger to the selected battery. Likewise, the discharge current of battery 2 is routed to the load system. The implemented system includes controlling and monitoring power management of charging and discharging process through solar PV panel.

\section{e. UART}

UART is an Universal Asynchronous Receiver and Transmitter. It's used for an translate the data between serial and parallel. In order to an transfer the data between transmitter and receiver. The input takes for bytes of data and transmitter and receiver the individual bits. It is used for a same bit speed, Character length, parity start bit and stop bit.

\section{Simulation Result:}

The robotic application has been processed to produce the simulation output for an Battery control, Light intensity, Robot movement. And in order to find for humidity and Temperature. For this two UART has used for produced the serial output. For the robot has moved for an F, B, L, R for its Four Movement. The robot used for an ASCII codes.

The ASCII codes contains for an 8 bit serial data input. The serial input has to given for an UART communication line. The clock input value is set for a rising edge. The reset value is changed for to give a shift register value. 


\begin{tabular}{|l|l|l|}
\hline Movement & ASCII Codes & Rotating Output \\
\hline Forward & 01000101 & $\mathrm{I} 1, \mathrm{i} 3$ \\
\hline Backward & 01000010 & $\mathrm{I} 2, \mathrm{i} 4$ \\
\hline Left & 01001100 & $\mathrm{I} 1, \mathrm{i} 2$ \\
\hline Right & 01010010 & $\mathrm{I} 3, \mathrm{i} 4$ \\
\hline
\end{tabular}

Table.1 ASCII values for Robot Movement

A/D converter translates an analog signal into a digital value. An 8 channel, 8-bit A/D input is available to read analog voltages between 0 to 5 Volts. .

When a RX input value has given the values to one to one after receiving the 8 input values the Robot movement direction has changed. The table shows for an ASCII code of robot movement.

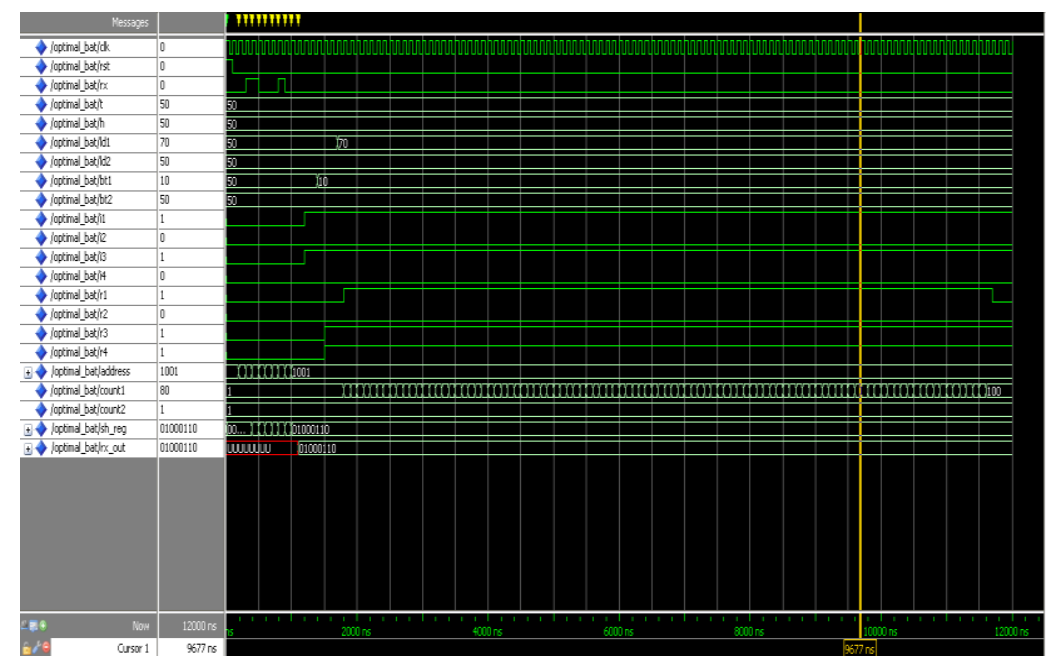

Fig.4 Simulation result of Full system VHDL code

The output sensor ld1 and ld2 has connected to Solar panels. These sensors are connected to the two sides it is checks for an light intensity and it compared to two sensors. In which contain high value rover has changed. The relay $\mathrm{r} 1, \mathrm{r} 2$ has changed.

At last the battery line b1, b2 has connected to the measuring unit its connected to the solar panel and to the robot. The battery value has stored. When a battery value is low it's automatically switched to solar panel. Another line has switch to robot.

\section{Conclusion:}

The energy management system applied to a robotic platform called VANTER devoted to exploration task and the system energy increased by the solar tracking based on PV panels. The amount of generated power independent from rover mobility.Hence the proposed mechanism capable of tracking maximum power. The development of VHDL code integrates optimal battery charging mechanism by implementing the dual battery pack and solar tracker and robot movement. The simulation results are verified by Model Sim.

\section{Reference}

[1]. de J Mateo Sanguino, T.; Gonzalez Ramos, J.E., "Smart Host Microcontroller for Optimal Battery Charging in a Solar-Powered Robotic Vehicle," Mechatronics, IEEE/ASME Transactions on , vol.18, no.3, pp.1039,1049, June 2013.

[2]. Hsu-Chih Huang; Ching-Chih Tsai, "FPGA Implementation of an Embedded Robust Adaptive Controller for Autonomous Omnidirectional Mobile Platform," Industrial Electronics, IEEE Transactions on, vol.56, no.5, pp.1604, 1616, May 2009.

[3]. Masoum, M. A S; Badejani, S.M.M.; Fuchs, E.F., "Microprocessor-controlled new class of optimal battery chargers for photovoltaic applications," Power Engineering Society General Meeting, 2004. IEEE, vol., no., pp.1489 Vol.2,, 10-10 June 2004.

[4]. Koutroulis, E.; Kalaitzakis, K.; Voulgaris, N.C., "Development of a microcontroller-based, photovoltaic maximum power point tracking control system," Power Electronics, IEEE Transactions on, vol.16, no.1, pp.46,54, Jan 2001.

[5]. Zhihua Qu; Jing Wang; Plaisted, C.E., "A new analytical solution to mobile robot trajectory generation in the presence of moving obstacles," Robotics, IEEE Transactions on , vol.20, no.6, pp.978,993, Dec. 2004.

[6]. Kimberly Shillcutt; William Whittaker., "Solar Navigational Planning for Robotic Explorers", Shillcutt and Whittaker, ICRA-2001.

[7]. Max Bajracharya, Mark W. Maimone, and Daniel Helmick, "Autonomy for Mars Rovers: Past, Present, and Future", Published by the IEEE Computer Society, 2008 IEEE. 
[8]. S.Michaud, A. Schneider, Bertrand, P.Lamon, R.Siegwart, M.Van Winnendael, A. Schiele, "solero: solar-powered exploration rover," ESA Workshop on Advanced Space Technologies for Robotics and Automation 'ASTRA 2002'ESTEC, Noordwijk, The Netherlands, November $19-21,2002$.

[9]. Theerawut Jinayim, Somchai runrungrasmi, Tanes Tanitteerapan, and Narong Mungkung., "Highly Efficient Low Power Consumption Tracking Solar Cells for White LED-Based Lighting System", World Academy of Science, Engineering and Technology International Journal of Electrical, Electronic Science and Engineering Vol:1 No:4, 2007.

Author Profile

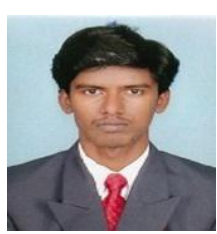

M.Ragulkumar, Post Graduate student- ME in VLSI Design at PGP College of Engineering and Technology, Namakkal.

P.Mankandan, Assistant Professor / Electronics and communication Engineering Department at PGP College of Engineering and Technology, Namakkal, Tamilnadu, India.

Dr.G.K.D.Prasanna Venkatesan, Completed Ph.D from College of Engineering, Anna University, Chennai, India. He is Currently Working as Vice-Principal, Professor \& Head of Department of Electronics and Communication Engineering at PGP College of Engineering and Technology. Namakkal, Tamil nadu, India. His research interests includes Wireless Sensor Networks, 4G Wireless Networks, Cloud Computing,Adhoc Network, etc., 